\title{
Two new species of the genus Betacixius Matsumura, 1914 from China (Hemiptera, Fulgoromorpha, Cixiidae)
}

\author{
Yan Zhi ${ }^{1,2}$, Chang-Hua Zhang ${ }^{4}$, Lin Yang ${ }^{1,3}$, Xiang-Sheng Chen ${ }^{1,3}$ \\ I Institute of Entomology, Guizhou University, Guiyang, Guizhou, 550025, China 2 Laboratory Animal \\ Center, Guizhou Medical University, Guiyang, Guizhou, 550025, China 3 The Provincial Special Key \\ Laboratory for Development and Utilization of Insect Resources of Guizhou, Guizhou University, Guiyang, \\ Guizhou, 550025, China 4 Guizhou Tobacco Company Zunyi Branch, Zunyi, Guizhou, 563000, China \\ Corresponding author: Xiang-Sheng Chen (chenxs3218@163.com)
}

Academic editor: M. Wilson | Received 16 January 2020 | Accepted 1 June 2020 | Published 6 August 2020

http://zoobank.org/AEA50D78-4B01-4A91-BBDB-9418E4CC2EA1

Citation: Zhi Y, Zhang C-H, Yang L, Chen X-S (2020) Two new species of the genus Betacixius Matsumura, 1914 from China (Hemiptera, Fulgoromorpha, Cixiidae). ZooKeys 956: 1-18. https://doi.org/10.3897/zookeys.956.50195

\begin{abstract}
Two new species of Betacixius Matsumura, 1914 (Fulgoromorpha, Cixiidae), B. latissimus Zhi \& Chen, sp. nov. and B. maguanensis Zhi \& Chen, sp. nov., are described from Yunnan Province, China. The genus is reviewed and a key to all known species is provided. The females of four Chinese species are described for the first time.
\end{abstract}

\section{Keywords}

Female genitalia, Fulgoroidea, morphology, Oriental region, taxonomy

\section{Introduction}

Betacixius is a small genus established by Matsumura (1914) for 23 species in the subfamily Cixiinae (Hemiptera, Fulgoromorpha, Cixiidae), with B. ocellatus Matsumura, 1914 as the type species. Betacixius belongs to the tribe Semonini (Emeljanov 2002) and has been taxonomically studied by various authors, with a number of species published successively (e.g. Jacobi 1944; Fennah 1956; Hori 1982; Tsaur et al. 1991; Zhang and Chen 2011).

Copyright Yan Zhi et al. This is an open access article distributed under the terms of the Creative Commons Attribution License (CC BY 4.0), which permits unrestricted use, distribution, and reproduction in any medium, provided the original author and source are credited. 
Recent study of some Chinese specimens has found two new species, B. latissimus Zhi \& Chen, sp. nov. and B. maguanensis Zhi \& Chen, sp. nov., which are described here. Female specimens of four species are also described for the first time. So far, including the two new species, the genus currently now counts for 25 valid species and two subspecies, all distributed in the Palaearctic and Oriental regions (Bourgoin 2020).

\section{Materials and methods}

The morphological terminology and measurements follow Bourgoin (1987) and Bourgoin et al. (2015). The morphological terminology of female genitalia follows Bourgoin (1993). Body length was measured from apex of vertex to tip of forewing; vertex length was measured the median length of vertex (from apical transverse carina to tip of basal emargination). Fuchsin staining was used to highlight female genitalia structures studied. External morphology and drawings were made with the aid of a Leica MZ 12.5 stereomicroscope. Photographs were taken with KEYENCE VHX-1000 system. Illustrations were scanned with a CanoScan LiDE 200 flatbed scanner and imported into Adobe Photoshop 7.0 for labeling and figure composition. The distribution map was generated with Google Earth Pro v. 7.3.2 (Google LLC). The dissected male and female genitalia are preserved in glycerin in small plastic tubes pinned together with the specimens.

The type specimens are deposited in the Institute of Entomology, Guizhou University, Guiyang, Guizhou Province, China (GUGC).

\section{Taxonomy}

\section{Betacixius Matsumura, 1914}

Betacixius Matsumura, 1914: 412; Tsaur et al. 1991: 27; Zhang and Chen 2011.

Type species. Betacixius ocellatus Matsumura, 1914, by original designation.

Diagnosis. See Zhang and Chen (2011: 48).

Distribution. China, Japan, Vietnam.

\section{Key to species of Betacixius}

1 Forewing with markings 2

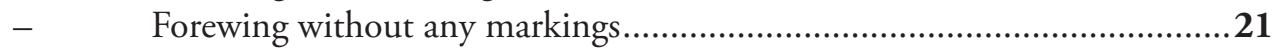

$2 \quad$ Forewing with a large ocellate marking in apical half .................................. 3

- $\quad$ Forewing without ocellate marking in apical half ........................................6

* revised from Fennah 1956; Zhang and Chen 2011 
Forewing with an oblique, brown band extending from clavus across middle of corium B. tonkinensis Matsumura, 1914

Forewing without such a band

Endosoma of aedeagus with one spine, hook-shaped (Zhang and Chen 2011: figs 22, 23). B. flagellihamus Zhang \& Chen, 2011 Endosoma of aedeagus with two spines, not hook-shaped.. . .5

B. maculosus Tsaur \& Hsu, 1991

Periandrium of aedeagus apically with one nearly straight and one arched processes

B. ocellatus Matsumura, 1914

Forewing with an oblique band extending from stigma passing through its middle part

Forewing with apical cells of $\mathrm{M}$ and $\mathrm{Cu}$ strongly infuscate......

B. transversus Jacobi, 1944

Forewing with apical cells not infuscate

Forewing with apical margin black or distinctly darkened....

Forewing with apical margin fuscous or not distinctly darkened

Frons with a pallid spot at centre of lateral margins; mesonotum testaceous....

B. kumejimae Matsumura, 1914

Frons without such spots; mesonotum, except scutellum, castaneous-piceous....

B. euterpe Fennah, 1956

Forewing with a spot near sutural margin of clavus near union of claval veins, no oblique dark band at this level extending into corium....

Forewing with an oblique dark band extending from clavus into centre of corium, slightly distad of level of union of claval veins

Forewing basally with a broad transverse band from dorsal margin to sutural margin of clavus (Fig. 3E)

B. latissimus sp. nov. Forewing without above band

B. obliquus Matsumura, 1914 Forewing basally with a light brown band..... B. pallidior Jacobi, 1944 Forewing basally without band B. michioi Hori, 1982 Forewing with a long black stripe from base, along clavus extending to Rs.... B. fuscus Tsaur \& Hsu, 1991

Forewing without such a stripe . .14 Forewing along the $\mathrm{R}$ with a black stripe widened towards the rear.

B. robustus Jacobi, 1944

Forewing without such a stripe

Ventral margin of periandrium basally with two broad, lobate processes (Zhang and Chen 2011: figs 10, 11)...... B. bispinus Zhang \& Chen, 2011 Ventral margin of periandrium basally without process .............................17 
18 Spinose process on right side of periandrium medium-sized, curved upwards, apex dorsally directed; spinose process on left side parallel to periandrium for most potion, apex ventrocephalically directed

B. rinkibonis Matsumura, 1914

- $\quad$ Spinose process on right side of periandrium very short, nearly straight, apex directed cephalad; spinose process on left side generally dorsocephalically directed

B. shirozui Hori, 1982

19 In lateral view, apical lobe of anal segment ventrally rounded.

B. delicatusTsaur \& Hsu, 1991

- In lateral view, apical lobe of anal segment ventrally pointed

20 Spinose process on right side of periandrium near dorsal margin, coiled 90 degrees to left; endosoma with two spinose processes

B. sparsus Tsaur \& Hsu, 1991

- $\quad$ Spinose process on right side of periandrium near ventral margin, nearly straight, apex directed cephalad; endosoma with one spinose process

B. maguanensis sp. nov.

21 Endosoma of aedeagus apically with two processes............................22

- $\quad$ Endosoma of aedeagus apically with one process..................................23

22 Ventral margin of periandrium with a long process

B. flavovittatus Hori, 1982

- Ventral margin of periandrium without process

23 Frons without median carina

B. nigromarginalis Fennah, 1956

Frons without median carin
Frons with median carina

B. clypealis Matsumura, 1914

Body pale brown; periandrium of aedeagus with two processes on right side....

B. brunneus Matsumura, 1914

Body green; periandrium of aedeagus with one process on each side

B. herbaceus Tsaur \& Hsu, 1991

\section{Betacixius bispinus Zhang \& Chen, 2011}

Figure 1A-I

Betacixius bispinus Zhang \& Chen, 2011: 53, figs 1-13, 24.

Material examined. China: $1 \hat{\jmath}$, Guizhou Province, Yanhe County, Mayanghe National Natural Reserve (600 m) (28 40'N, 108 19'E), 5-12 June 2007, Xiang-Sheng Chen (holotype); $1 \delta^{\Uparrow} 7$ 우, Guizhou Province, Yanhe County, Mayanghe National Natural Reserve (600 m), 5-12 June 2007, Xiang-Sheng Chen (paratypes); $1 \delta^{\lambda} 1$, Guizhou Province, Tongren City, Fanjingshan National Natural Reserve (500-1800 m)

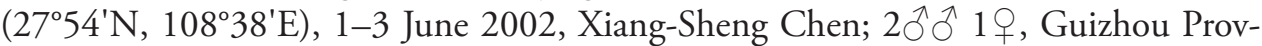
ince, Daozhen County, Sanqiao Town (29 $\left.3^{\prime} \mathrm{N}, 107^{\circ} 30^{\prime} \mathrm{E}\right), 24$ May 2002, XiangSheng Chen; $1 \delta^{\top}$, Guizhou Province, Chishui City, Shizhangdong Waterfall $\left(28^{\circ} 22^{\prime} \mathrm{N}\right.$, 

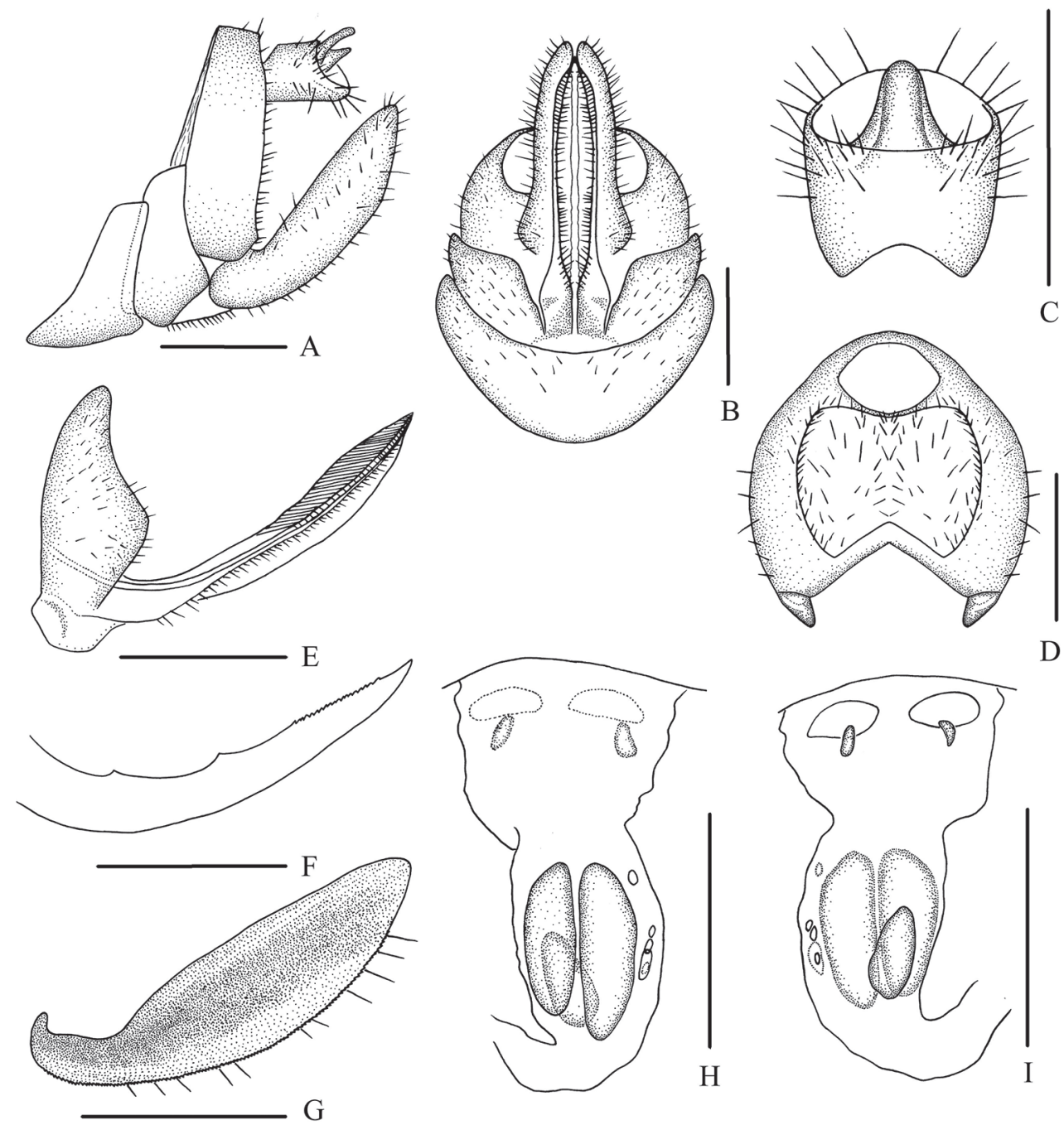

$\mathrm{D}$

Figure I. Betacixius bispinus Zhang \& Chen, 2011, female A genitalia, lateral view B genitalia, ventral view $\mathbf{C}$ anal segment, dorsal view D tergite IX, caudal view E gonapophysis VIII and gonocoxa VIII, ventral view $\mathbf{F}$ gonapophysis IX, lateral view $\mathbf{G}$ gonoplac, inner lateral view $\mathbf{H}$ posterior vagina, ventral view I posterior vagina, dorsal view. Scale bars: $0.5 \mathrm{~mm}$.

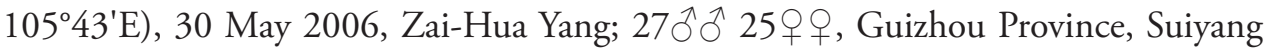
County, Kuankuoshui National Natural Reserve $\left(28^{\circ} 14^{\prime} \mathrm{N}, 107^{\circ} 12^{\prime} \mathrm{E}\right)$, 8-9 June

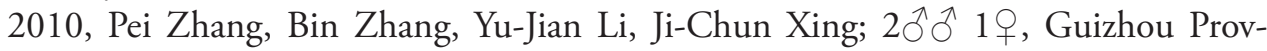
ince, Huishui County, Duanshan Town, Guangrong Village (2550'N, $\left.106^{\circ} 37^{\prime} \mathrm{E}\right), 9$ May 2013, Jian-Kun Long, Zai-Hua Yang; $1 \jmath_{1}$, Sichuan Province, Nanchong City, Dayou Township (3048'N, 106 41'E), 9-10 May 2008, Zheng-Guang Zhang, Zai-

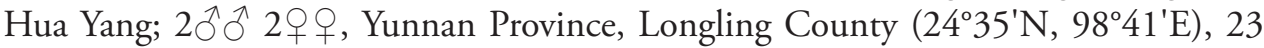

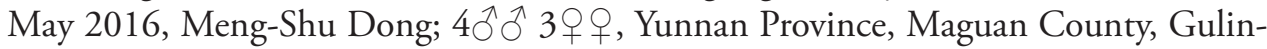


qing Township (22 $\left.48^{\prime} \mathrm{N}, 103^{\circ} 57^{\prime} \mathrm{E}\right), 30-31$ May 2016, Liang-Jing Yang, Qiang Luo, Ying-Jian Wang.

Supplementary description. Female genitalia. Tergite IX (Fig. 1A, B, D) moderately sclerotized, with a large nearly dumbbell-shaped wax plate. Anal segment (Fig. 1C) rectangular, widening to apex, 1.1 times wider than long in dorsal view, anal style strap-like. Gonapophysis VIII (Fig. 1E) elongate, and slightly curved upwards. Gonapophysis IX (Fig. 1F) with two middle teeth, distance ratio between distal middle tooth to apex and length of denticulate portion is 2.2. Gonoplac (Fig. 1G) rodlike, 3.5 times longer than wide in lateral view. Posterior vagina pattern as shown in Figure $1 \mathrm{H}, \mathrm{I}$.

Distribution. China (Guizhou, Sichuan, Yunnan).

Note. The female genitalia of this species are described and illustrated for the first time.

\section{Betacixius flagellihamus Zhang \& Chen, 2011}

Figure 2A-I

Betacixius flagellihamus Zhang \& Chen, 2011: 54, figs 14-23, 25.

Material examined. China: $1 \hat{\partial}$, Guizhou Province, Leishan County, Leigongshan National Natural Reserve $\left(26^{\circ} 31^{\prime} \mathrm{N}, 108^{\circ} 17^{\prime} \mathrm{E}\right), 13$ May 1985, Zi-Zhong Li (holotype); $7 \hat{\jmath} \widehat{0} 9$ 우우, Guizhou Province, Leishan County, Leigongshan National Natural Reserve, 13 May 1985, Zi-Zhong Li (paratypes); $2 \hat{\jmath} \hat{\jmath}$, Guizhou Province, Guiyang City, June 1983, Students of Grade 79, Major Plant Protection (paratypes); $1 \delta^{\Uparrow} 2$ + 9 , Guizhou Province, Guiyang City, Forest Park (1000 m) (26 32'N, 106 $\left.45^{\circ} \mathrm{E}\right), 20$ May 2007, Xiang-Sheng Chen (paratypes); $1 \delta^{\Uparrow} 1$, Guizhou Province, Guiyang City, Forest Park, 14-17 May 1984, collector unknown; $2 \hat{\jmath} 0^{\lambda} 1$, Guizhou Province, Duyun City, Gantang Town, Tuanzhai Village $\left(26^{\circ} 16^{\prime} \mathrm{N}, 107^{\circ} 26^{\prime} \mathrm{E}\right), 12$ May 2014, Ming Ning, Gai-Ping Yang; $1 \delta^{\lambda} 5 ㅇ+$, Guizhou Province, Suiyang County, Kuankuoshui National Natural Reserve $\left(28^{\circ} 14^{\prime} \mathrm{N}, 107^{\circ} 12^{\prime} \mathrm{E}\right)$, 8-9 June 2010, Pei Zhang, Bin Zhang;

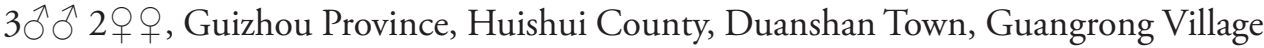
$\left(25^{\circ} 50^{\prime} \mathrm{N}, 106^{\circ} 37^{\prime} \mathrm{E}\right), 9$ May 2013, Jian-Kun Long, Zai-Hua Yang; $10^{\wedge} 3 q 9$, Guizhou Province, Guiyang City, Huaxi District $\left(26^{\circ} 25^{\prime} \mathrm{N}, 106^{\circ} 40^{\prime} \mathrm{E}\right), 15-16$ May 1982, col-

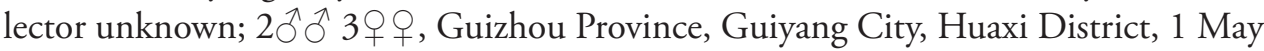

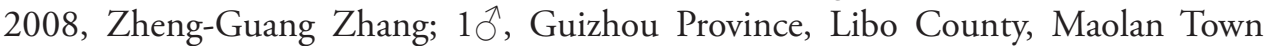

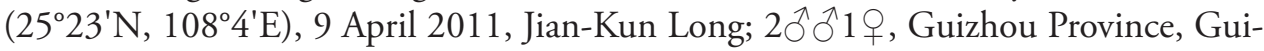
yang City, Forest Park, 24 May 2006, Zai-Hua Yang; $3 \hat{\jmath} \widehat{\jmath} 2$ 우 $ᄋ$, Guizhou Province, Guiyang City, Forest Park, 21 April 2010, Jun-Qiang Ni; 10 đิ $^{\widehat{T}} 6$ 6우, Guizhou Prov-

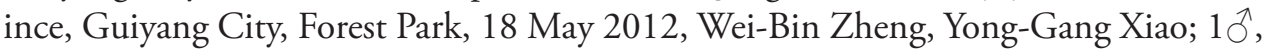
Guizhou Province, Guiyang City, Huaxi District, 29 April 2017, Yong-Jin Sui.

Supplementary description. Female genitalia. Tergite IX (Fig. 2A, B, D) moderately sclerotized, with a large nearly dumbbell-shaped wax plate. Anal segment (Fig. 2C) rectangular, widening to apex, 1.5 times wider than long in dorsal view, 


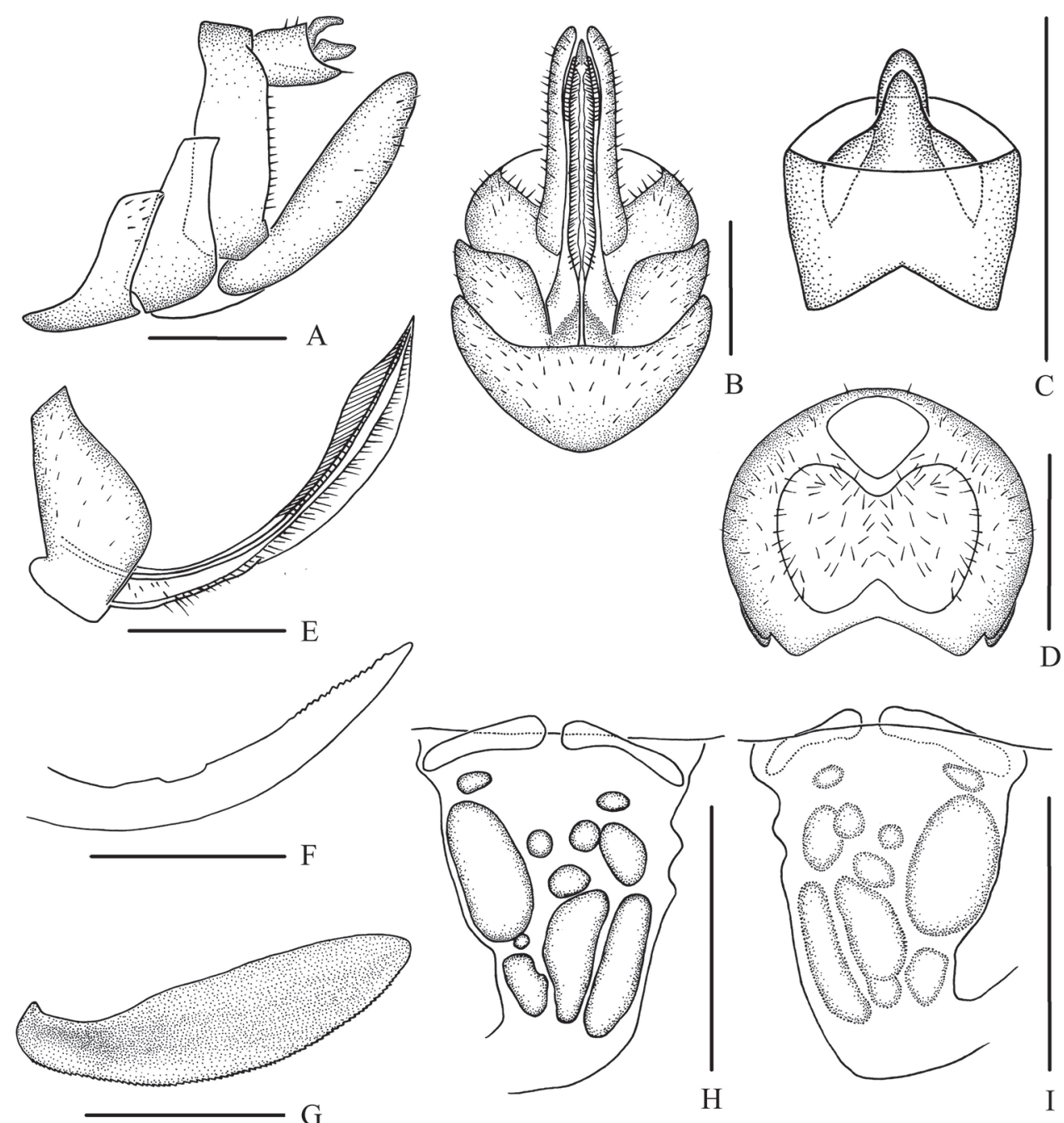

Figure 2. Betacixius flagellihamus Zhang \& Chen, 2011, female A genitalia, lateral view B genitalia, ventral view $\mathbf{C}$ anal segment, dorsal view $\mathbf{D}$ tergite IX, caudal view $\mathbf{E}$ gonapophysis VIII and gonocoxa VIII, ventral view $\mathbf{F}$ gonapophysis IX, lateral view $\mathbf{G}$ gonoplac, inner lateral view $\mathbf{H}$ posterior vagina, ventral view I posterior vagina, dorsal view. Scale bars: $0.5 \mathrm{~mm}$.

anal style strap-like. Gonapophysis VIII (Fig. 2E) elongate, and slightly curved upwards. Gonapophysis IX (Fig. 2F) with two middle teeth, distance ratio between distal middle tooth to apex and length of denticulate portion is 2.1. Gonoplac (Fig. 2G) rod-like, 3.6 times longer than wide in lateral view. Posterior vagina pattern as shown in Figure 2H, I.

Host plant. Quercus sp. (Fagaceae).

Distribution. China (Guizhou).

Note. The female genitalia of this species are described and illustrated for the first time. 


\section{Betacixius latissimus sp. nov.}

http://zoobank.org/ED00F996-CE3B-4BAB-8B3A-C3427C482BC5

Figures 3A-M, 4A-I

Type material. Holotype: $\widehat{\alpha}$, China: Yunnan Province, Jinping County, Fenshuiling National Nature Reserve $\left(22^{\circ} 46^{\prime} \mathrm{N}, 103^{\circ} 13^{\prime} \mathrm{E}\right), 7-8$ June 2016, Liang-Jing Yang, YingJian Wang; paratypes: $10^{\lambda} 1$, same data as holotype.

Description. Body length: male 6.9-7.1 $\mathrm{mm}(n=2)$, female $7.2 \mathrm{~mm}(n=1)$.

Coloration. General color blackish brown (Fig. 3A-D). Eyes reddish brown, lateral ocelli dark red and median ocellus yellow. Vertex dark brown, pronotum yellowish to blackish brown and mesonotum blackish brown. Face generally dark brown, yellowish white above frontoclypeal suture. Postclypeus yellowish brown and anteclypeus blackish brown. Rostrum generally yellowish brown except darker tip. Forewing semitranslucent, base with a broad transverse band from dorsal margin to sutural margin of clavus; clavus with a blackish brown spot on apical third, an oblique transverse band originating from stigma extending to ventral margin. Hind tibiae yellowish brown and abdominal sternites blackish brown.

Head and thorax. Vertex (Fig. 3A, C) broad, 1.9 times wider than long; anterior margin arched convex, posterior margin slightly arched concave; median carina distinct and complete. Frons (Fig. 3D) 0.6 times as long as wide, median carina indistinct, extending from slightly above level of lateral ocelli to median ocellus. $\mathrm{Cl}$ ypeus with median carina distinct and elevated throughout. Pronotum (Fig. 3C) 1.4 times longer than vertex, posterior margin concaved in obtuse angle. Mesonotum 1.6 times longer than pronotum and vertex combined. Forewing (Fig. 3E) 1.9 times longer than wide, with nine apical and five subapical cells; fork $\mathrm{Sc}+\mathrm{RP}$ slightly distad of fork $\mathrm{CuA} 1+\mathrm{CuA2}$; first crossvein $\mathrm{r}-\mathrm{m}$ slightly distad of fork MP; RP 2 branches, MP with four terminals: MP 1, MP2, MP3, and MP4, fork MP1+MP2 almost at same level as fork MP3+MP4. Hind tibia with three lateral spines, six apical spines; chaetotaxy of hind tarsi: 7/7, second segment of hind tarsus with three platellae.

Male genitalia. Pygofer (Fig. 3F, G) symmetrical, dorsal margin concave and Ushaped ventrally, widened towards apex; in lateral view, lateral lobes arched extended caudally. Medioventral process triangular in ventral view. Anal segment (Fig. 3F, H) long, tubular, asymmetrical, apical lobes arched extended ventrally in lateral view, left lobe larger than right one, 2.8 times longer than wide in dorsal view; anal style fingerlike, not beyond anal segment. Gonostyli (Fig. 3F, G, I) symmetrical in ventral view; in inner lateral view, apical part extended, apical margin acute. Aedeagus (Fig. 3J-M) in total with four processes. Right side of periandrium with a long spinose process apically, straight for most portion, directed dorsocephalically, apex curved downwards and directed ventrocephalically; left side of periandrium apically with two medium-sized spinose processes, the upper one strongly curved and directed dorsally and the other one slightly curved and directed cephalad. Base of periandrium ventrally with several saw-like teeth. Endosoma slender, structure simple, apically with a small spinose process on left margin. 

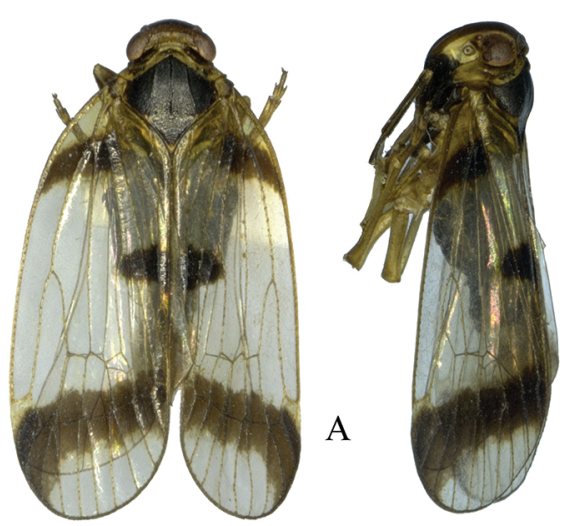

B
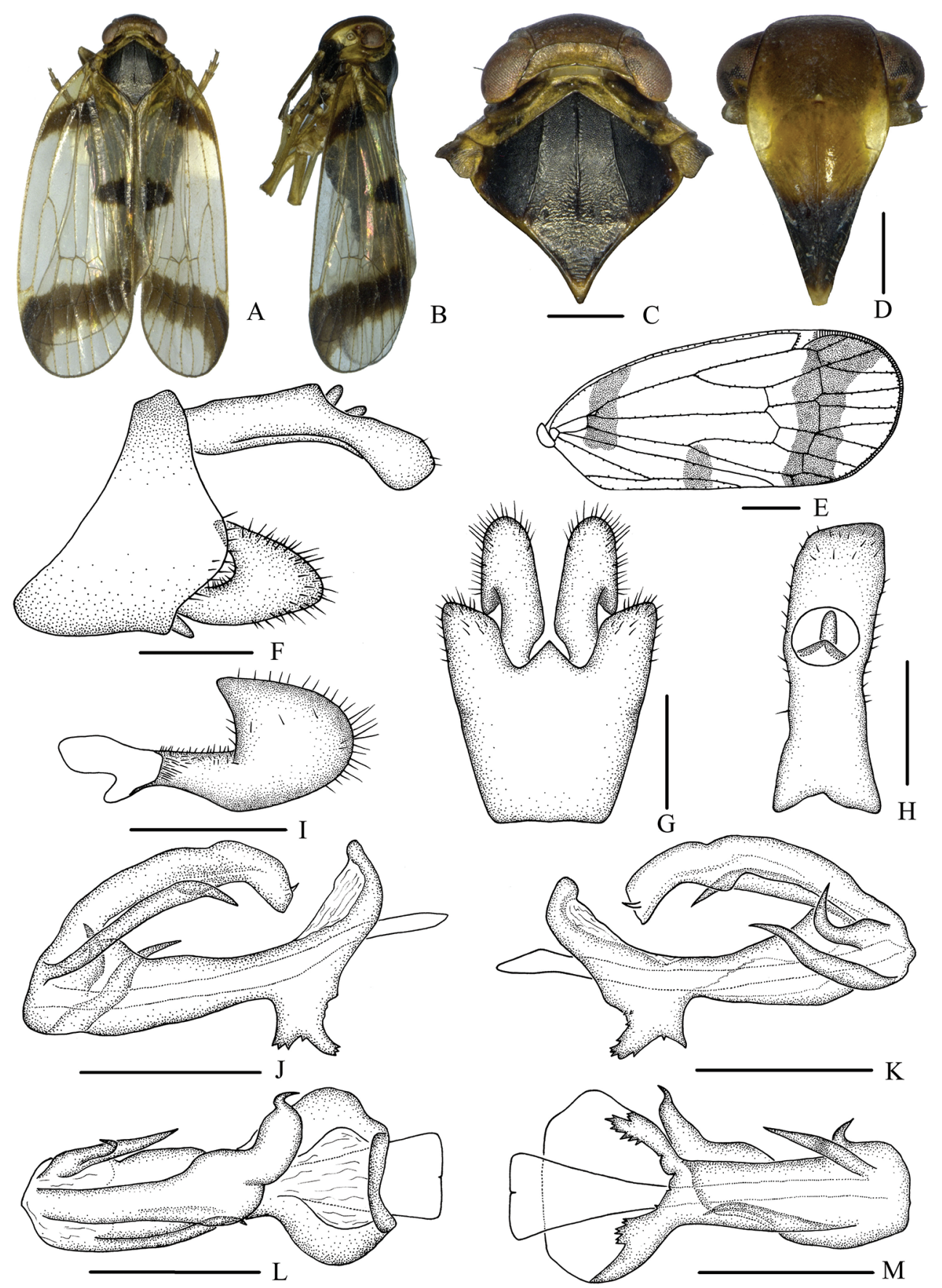

Figure 3. Betacixius latissimus sp. nov., male $\mathbf{A}$ habitus, dorsal view $\mathbf{B}$ habitus, lateral view $\mathbf{C}$ head and thorax, dorsal view $\mathbf{D}$ face, ventral view $\mathbf{E}$ forewing $\mathbf{F}$ genitalia, lateral view $\mathbf{G}$ pygofer and gonostyli, ventral view $\mathbf{H}$ anal segment, dorsal view $\mathbf{I}$ gonostyli, inner lateral view $\mathbf{J}$ aedeagus, right side $\mathbf{K}$ aedeagus, left side $\mathbf{L}$ aedeagus, dorsal view $\mathbf{M}$ aedeagus, ventral view. Scale bars: $0.5 \mathrm{~mm}(\mathbf{C}, \mathbf{D}, \mathbf{F}-\mathbf{M}) ; 1.0 \mathrm{~mm}(\mathbf{E})$. 

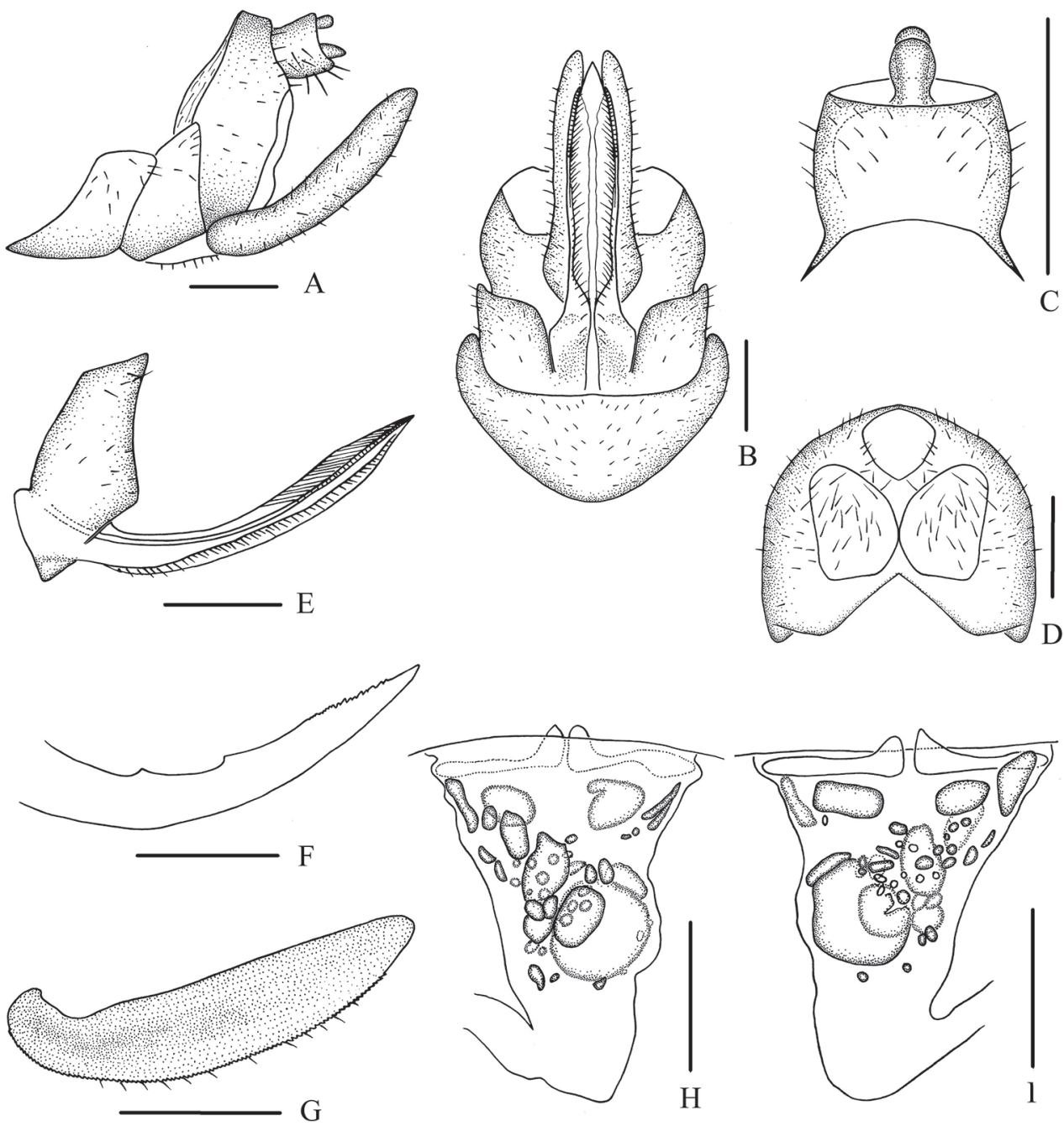

Figure 4. Betacixius latissimus sp. nov., female $\mathbf{A}$ genitalia, lateral view $\mathbf{B}$ genitalia, ventral view $\mathbf{C}$ anal segment, dorsal view D tergite IX, caudal view E gonapophysis VIII and gonocoxa VIII, ventral view $\mathbf{F}$ gonapophysis IX, lateral view $\mathbf{G}$ gonoplac, inner lateral view $\mathbf{H}$ posterior vagina, ventral view I posterior vagina, dorsal view. Scale bars: $0.5 \mathrm{~mm}$.

Female genitalia. Tergite IX (Fig. 4A, B, D) moderately sclerotized, with two connected wax plates, nearly oval. Anal segment (Fig. 4C) rectangular, 1.4 times wider than long in dorsal view, anal style strap-like. Gonapophysis VIII (Fig. 4E) elongate, and slightly curved upwards. Gonapophysis IX (Fig. 4F) with two middle teeth, distance ratio between distal middle tooth to apex and length of denticulate portion is 2.4. Gonoplac (Fig. 4G) rod-like, 3.8 times longer than wide in lateral view. Posterior vagina pattern as shown in Figure 4H, I.

Distribution. China (Yunnan). 
Etymology. The specific name, derived from Latin word meaning the broadest, refers to the forewing with an extremely broad band at apex.

Remarks. Male genitalia of $B$. latissimus sp. nov. is similar to B. herbaceus Tsaur \& Hsu, 1991, but differs in: (1) left side of periandrium with two spinose processes (in $B$. herbaceus, left side of periandrium with one spinose process); (2) spinose process on right of periandrium curved downwards (in $B$. herbaceus, spinose process in the same position curved upwards); (3) anal segment asymmetrical (the latter symmetrical); (4) forewing with one blackish brown spot and two bands (the latter without any marking).

\section{Betacixius maculosus Tsaur \& Hsu, 1991}

Figure 5A-I

Betacixius maculosus Tsaur and Hsu in Tsaur et al. 1991: 31, fig. 15A-I.

Material examined. China: $3 \hat{\partial} \sigma^{\lambda} 1$, Taiwan Province, Nantou County $\left(24^{\circ} 2^{\prime} \mathrm{N}\right.$, $\left.121^{\circ} 7^{\prime} \mathrm{E}\right), 25$ November 2002, Xiang-Sheng Chen.

Supplementary description. Female genitalia. Tergite IX (Fig. 5A, B, D) moderately sclerotized, with a medium-sized, nearly dumbbell-shaped wax plate. Anal segment (Fig. 5C) rectangular, slightly widening to apex, 1.1 times longer than wide in dorsal view, anal style finger-like. Gonapophysis VIII (Fig. 5E) elongate, and slightly curved upwards. Gonapophysis IX (Fig. 5F) with two middle teeth, distance ratio between distal middle tooth to apex and length of denticulate portion is 2.1. Gonoplac (Fig. 5G) rod-like, 3.4 times longer than wide in lateral view. Posterior vagina pattern as shown in Figure 5H, I.

Distribution. China (Taiwan).

Note. The female genitalia of this species are described and illustrated for the first time in detail.

\section{Betacixius maguanensis sp. nov.}

http://zoobank.org/2FF88233-4AAE-4F3E-9351-C5759FC12F4E

Figures 6A-M, 7A-I

Type material. Holotype: $\lambda$, China: Yunnan Province, Maguan County, Gulinqing Township (22 $\left.48^{\prime} \mathrm{N}, 103^{\circ} 57^{\prime} \mathrm{E}\right), 30-31$ May 2016, Liang-Jing Yang, Ying-Jian Wang, Qiang Luo; paratypes: $8 \widehat{o}^{\hat{\jmath}} 5$ 우우, same data as holotype.

Description. Body length: male 5.9-6.5 mm $(n=9)$, female 6.7-7.0 $\mathrm{mm}(n=5)$.

Coloration. General color blackish brown (Fig. 6A-D). Eyes reddish brown, ocelli light yellow. Vertex yellowish brown, pronotum yellowish brown and mesonotum black. Face generally yellowish brown, yellowish white above frontoclypeal suture. Postclypeus yellow and anteclypeus blackish brown. Rostrum generally yellowish brown. Forewing semi-translucent, clavus with a blackish brown spot on apical third, 

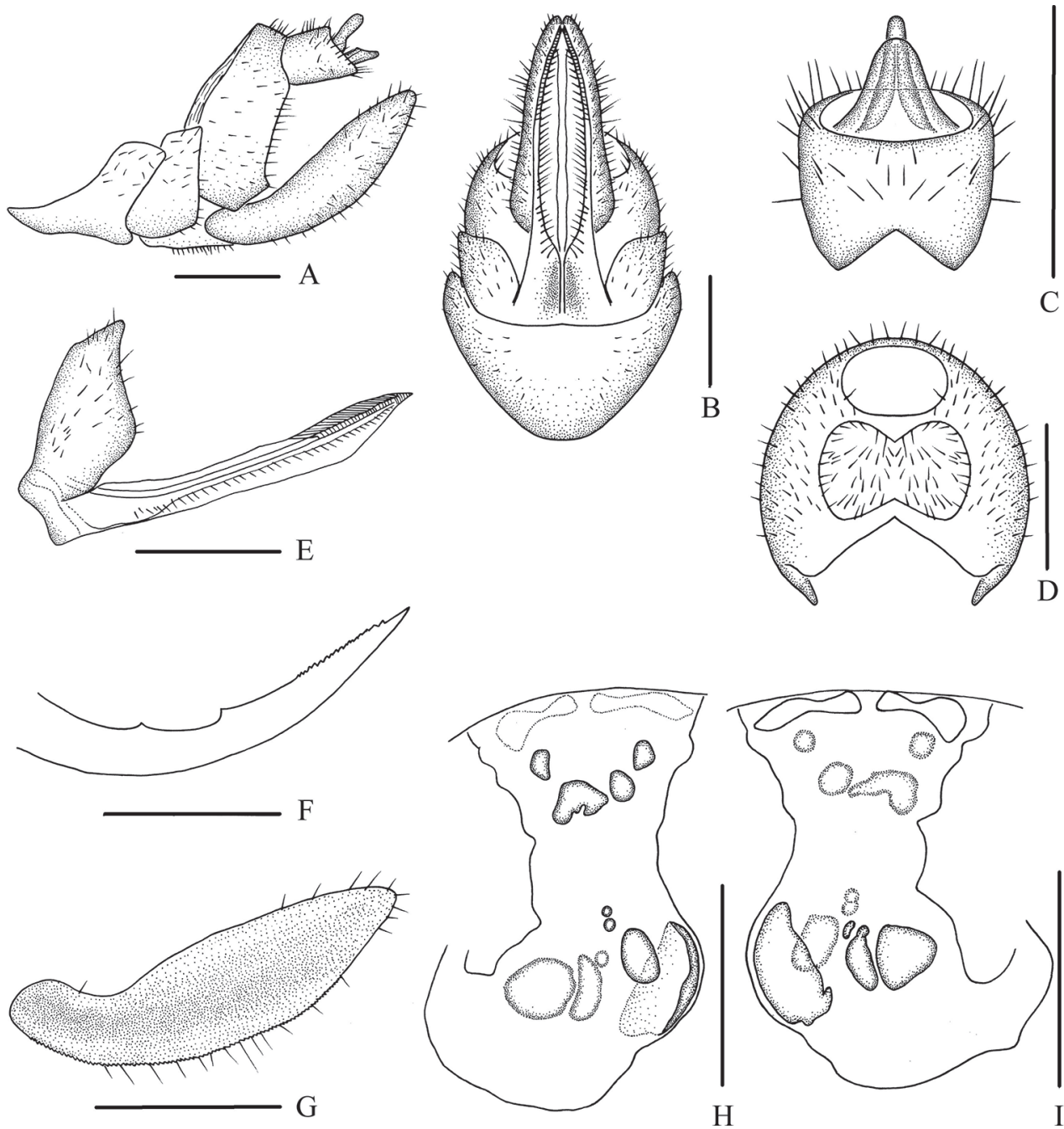

Figure 5. Betacixius maculosus Tsaur \& Hsu, 1991, female A genitalia, lateral view B genitalia, ventral view $\mathbf{C}$ anal segment, dorsal view $\mathbf{D}$ tergite IX, caudal view E gonapophysis VIII and gonocoxa VIII, ventral view $\mathbf{F}$ gonapophysis IX, lateral view $\mathbf{G}$ gonoplac, inner lateral view $\mathbf{H}$ posterior vagina, ventral view I posterior vagina, dorsal view. Scale bars: $0.5 \mathrm{~mm}$.

stigma blackish brown. Hind tibiae light blackish brown and abdominal sternites blackish brown.

Head and thorax. Vertex (Fig. 6A, C) broad, 2.6 times wider than long; anterior margin slightly arched convex, posterior margin arched concave; median carina distinct and complete. Frons (Fig. 6D) 0.8 times as long as wide, median carina indistinct, extending from slightly above level of lateral ocelli to median ocellus. Clypeus with median carina distinct and elevated throughout. Pronotum (Fig. 6C) 2.3 times longer than vertex, posterior margin concaved in an obtuse angle. Mesonotum 


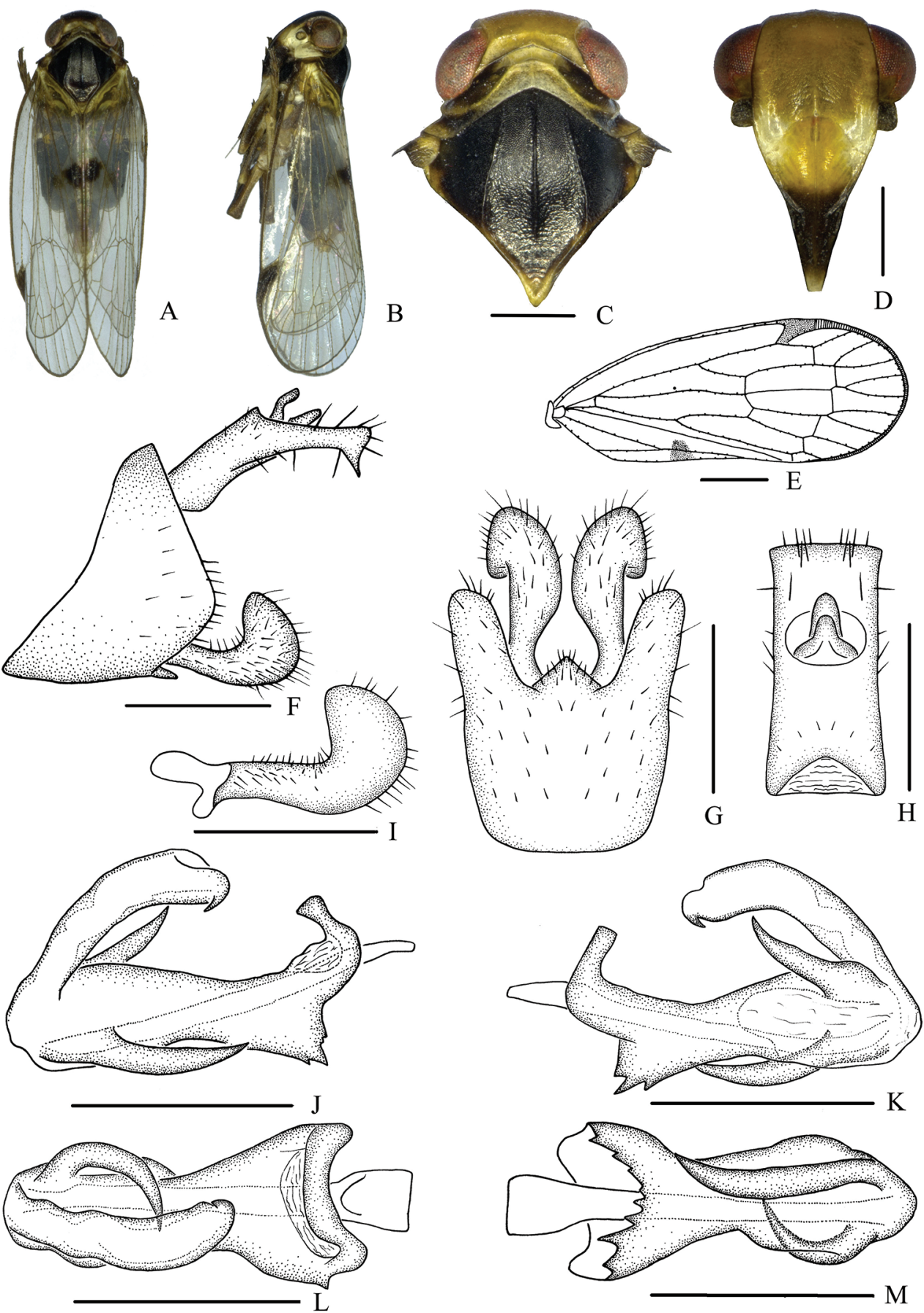

Figure 6. Betacixius maguanensis sp. nov., male A habitus, dorsal view $\mathbf{B}$ habitus, lateral view $\mathbf{C}$ head and thorax, dorsal view $\mathbf{D}$ face, ventral view $\mathbf{E}$ forewing $\mathbf{F}$ genitalia, lateral view $\mathbf{G}$ pygofer and gonostyli, ventral view $\mathbf{H}$ anal segment, dorsal view $\mathbf{I}$ gonostyli, inner lateral view $\mathbf{J}$ aedeagus, right side $\mathbf{K}$ aedeagus, left side $\mathbf{L}$ aedeagus, dorsal view $\mathbf{M}$ aedeagus, ventral view. Scale bars: $0.5 \mathrm{~mm}(\mathbf{C}, \mathbf{D}, \mathbf{F}-\mathbf{M}) ; 1.0 \mathrm{~mm}(\mathbf{E})$. 


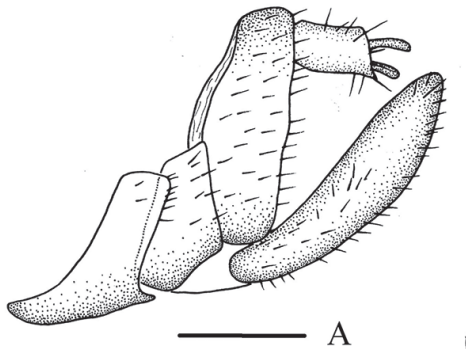

A
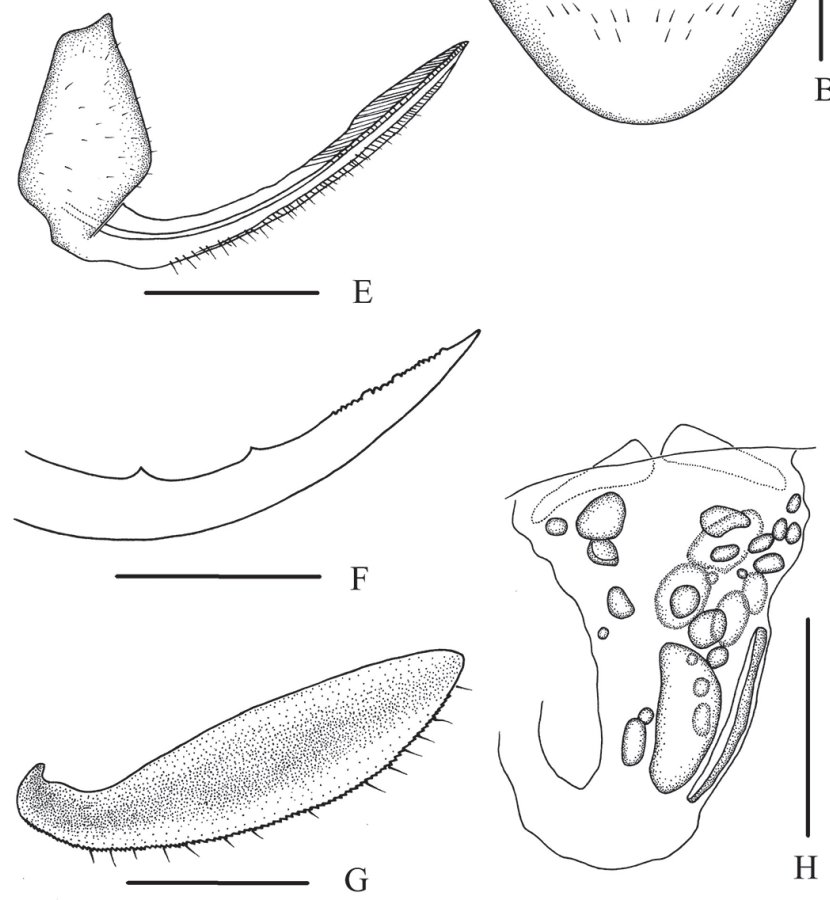

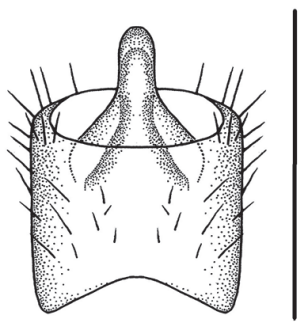

$\mathrm{C}$

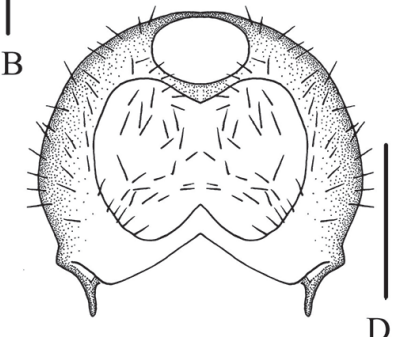

$\mathrm{D}$

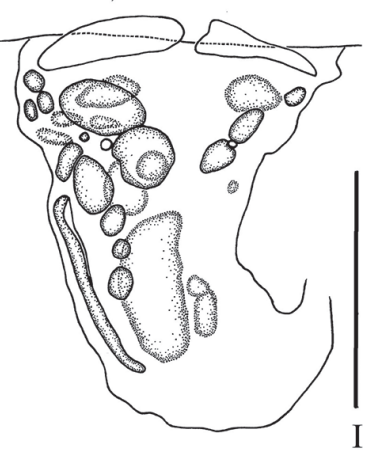

Figure 7. Betacixius maguanensis sp. nov., female $\mathbf{A}$ genitalia, lateral view B genitalia, ventral view $\mathbf{C}$ anal segment, dorsal view D tergite IX, caudal view E gonapophysis VIII and gonocoxa VIII, ventral view $\mathbf{F}$ gonapophysis IX, lateral view $\mathbf{G}$ gonoplac, inner lateral view $\mathbf{H}$ posterior vagina, ventral view $\mathbf{I}$ posterior vagina, dorsal view. Scale bars: $0.5 \mathrm{~mm}$.

1.7 times longer than pronotum and vertex combined. Forewing (Fig. 6E) 2.5 times longer than wide, with nine apical and five subapical cells; fork Sc+RP distad of fork $\mathrm{CuA1}+\mathrm{CuA2}$; first crossvein $\mathrm{r}-\mathrm{m}$ almost at same level as fork MP; RP 2 branches, MP with four terminals: MP 1, MP2, MP3, and MP4, fork MP1+MP2 distad of fork MP3+MP4. Hind tibia with three lateral spines, six apical spines; chaetotaxy of hind tarsi: 7/7, second segment of hind tarsus with three platellae.

Male genitalia. Pygofer (Fig. 6F, G) symmetrical, dorsal margin concave and U-shaped ventrally, widened towards apex; in lateral view, lateral lobes triangularly extended caudally. Medioventral process triangular in ventral view. Anal segment (Fig. 6F, H) long tubular, symmetrical, apical lobes ventrally pointed, 2.1 times longer 
than wide in dorsal view; anal style strap-like, not extending beyond anal segment. Gonostyli (Fig. 6F, G, I) symmetrical in ventral view; in inner lateral view, apical part extended, apical margin rounded. Aedeagus (Fig. 6J-M) with three processes. Right side near ventral margin of periandrium apically with a long spinose process, nearly straight, apex directed cephalad; left side near dorsal margin of periandrium with a shorter spinose process curving upwards, apex right-dorsally directed. Base of periandrium ventrally with several saw-like teeth. Endosoma slender, structure simple, apically with a small hook-like spinose process.

Female genitalia. Tergite IX (Fig. 7A, B, D) moderately sclerotized, with a large, nearly dumbbell-shaped wax plate. Anal segment (Fig. 7C) rectangular, 1.1 times longer than wide in dorsal view, anal style finger-like. Gonapophysis VIII (Fig. 7E) elongate, and slightly curved upwards. Gonapophysis IX (Fig. 7F) with two middle teeth, distance ratio between distal middle tooth to apex and length of denticulate portion is 2.0. Gonoplac (Fig. 7G) rod-like, 3.8 times longer than wide in lateral view. Posterior vagina pattern as shown in Figure $7 \mathrm{H}$, I.

Distribution. China (Yunnan).

Etymology. The species name is derived from Maguan County, Yunan Province, where the type locality is located.

Remarks. Male genitalia of B. maguanensis sp. nov. is similar to B. flagellihamus Zhang \& Chen, 2011, but differs in: (1) spinose process on right of periandrium nearly straight, directed cephalad (in B. flagellihamus, spinose process on right of periandrium curving dorsally); (2) apical lobes of anal segment pointed ventrally (the latter rounded); (3) forewing without ocellate marking (the latter with a large ocellate marking in apical half).

\section{Betacixius sparsus Tsaur \& Hsu, 1991}

Figure 8A-I

Betacixius sparsus Tsaur and Hsu in Tsaur et al. 1991: 46, fig. 23A-E.

Material examined. China: $20 \overbrace{}^{\lambda} 56 q q$, Guangxi Province, Wuming County, Daming Mountain $\left(23^{\circ} 27^{\prime} \mathrm{N}, 108^{\circ} 27^{\prime} \mathrm{E}\right), 14-15$ May 2012, Zhi-Hua Fan, Hu Li, Nan-nan

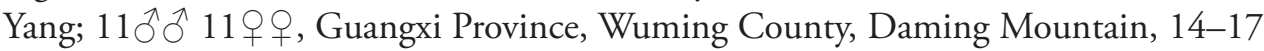
May 2011, Xiao-Fei Yu, Rong Huang, Xin-Feng Zhang; $4 \hat{\jmath} \widehat{o} 4 q$, ince, Shangsi County, Shiwandashan National Forest Park (21 $\left.{ }^{\circ} 56^{\prime} \mathrm{N}, 108^{\circ} 6^{\prime} \mathrm{E}\right), 2-4$ May 2011, Xiao-Fei Yu, Rong Huang.

Supplementary description. Female genitalia. Tergite IX (Fig. 8A, B, D) moderately sclerotized, with two nearly oval wax plates, separated from each other. Anal segment (Fig. 8C) rectangular, widening to apex, 1.1 times wider than long in dorsal view, anal style strap-like. Gonapophysis VIII (Fig. 8E) elongate, and slightly curved upwards. Gonapophysis IX (Fig. 8F) with two middle teeth, distance ratio between distal middle tooth to apex and length of denticulate portion is 2.3. Gonoplac (Fig. 8G) rod-like, 3.8 times longer than wide in lateral view. Posterior vagina pattern as shown in Figure 8H, I. 

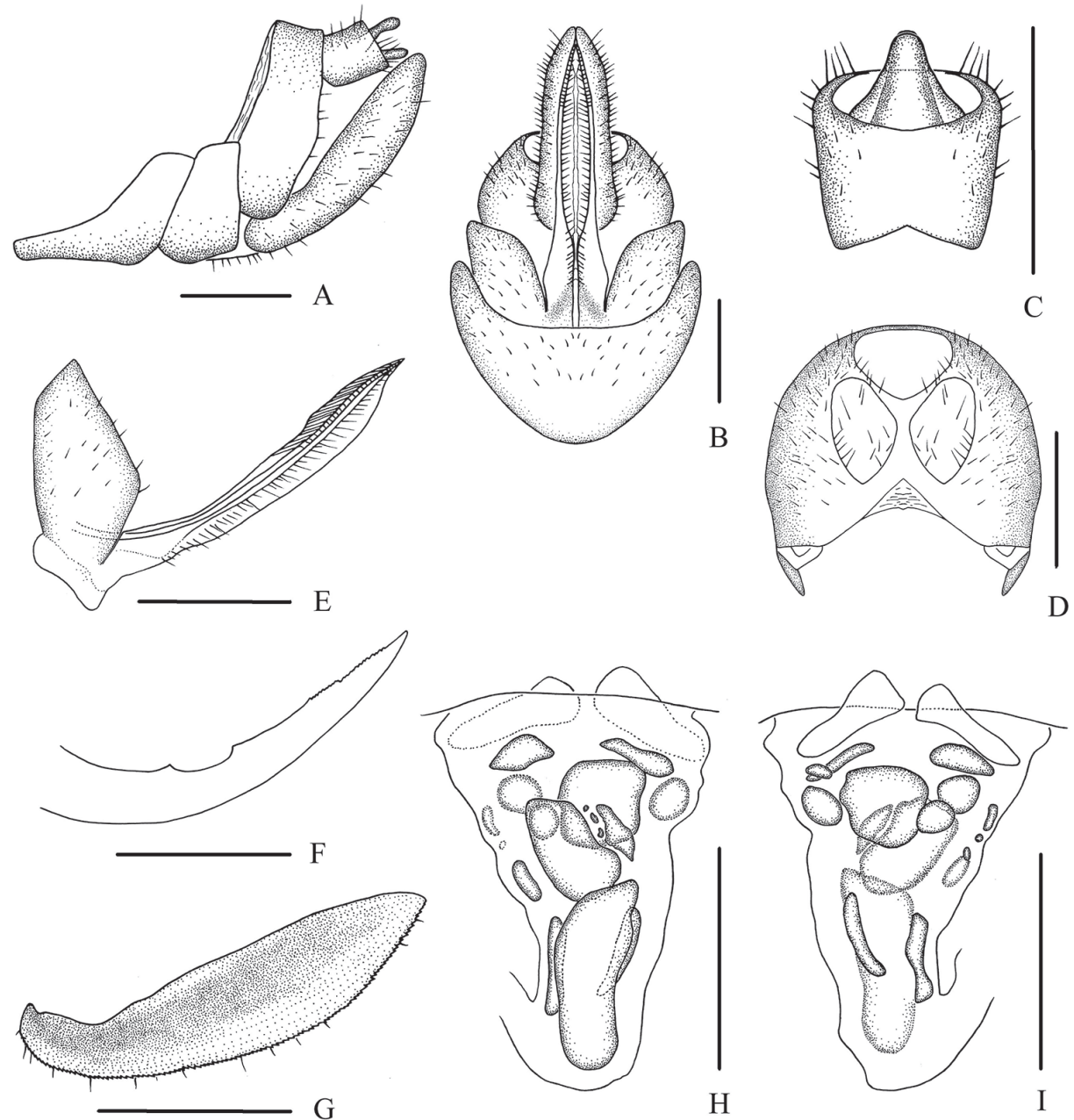

Figure 8. Betacixius sparsus Tsaur \& Hsu, 1991, female A genitalia, lateral view B genitalia, ventral view C anal segment, dorsal view D tergite IX, caudal view E gonapophysis VIII and gonocoxa VIII, ventral view $\mathbf{F}$ gonapophysis IX, lateral view $\mathbf{G}$ gonoplac, inner lateral view $\mathbf{H}$ posterior vagina, ventral view I posterior vagina, dorsal view. Scale bars: $0.5 \mathrm{~mm}$.

Distribution. China (Guangxi, Taiwan).

Note. The female genitalia of this species are described and illustrated for the first time.

\section{Discussion}

Prior to this study, nothing has been reported on the host plants of Betacixius. Nonetheless, we found these planthoppers most commonly in grass, shrubs, or on bamboo 


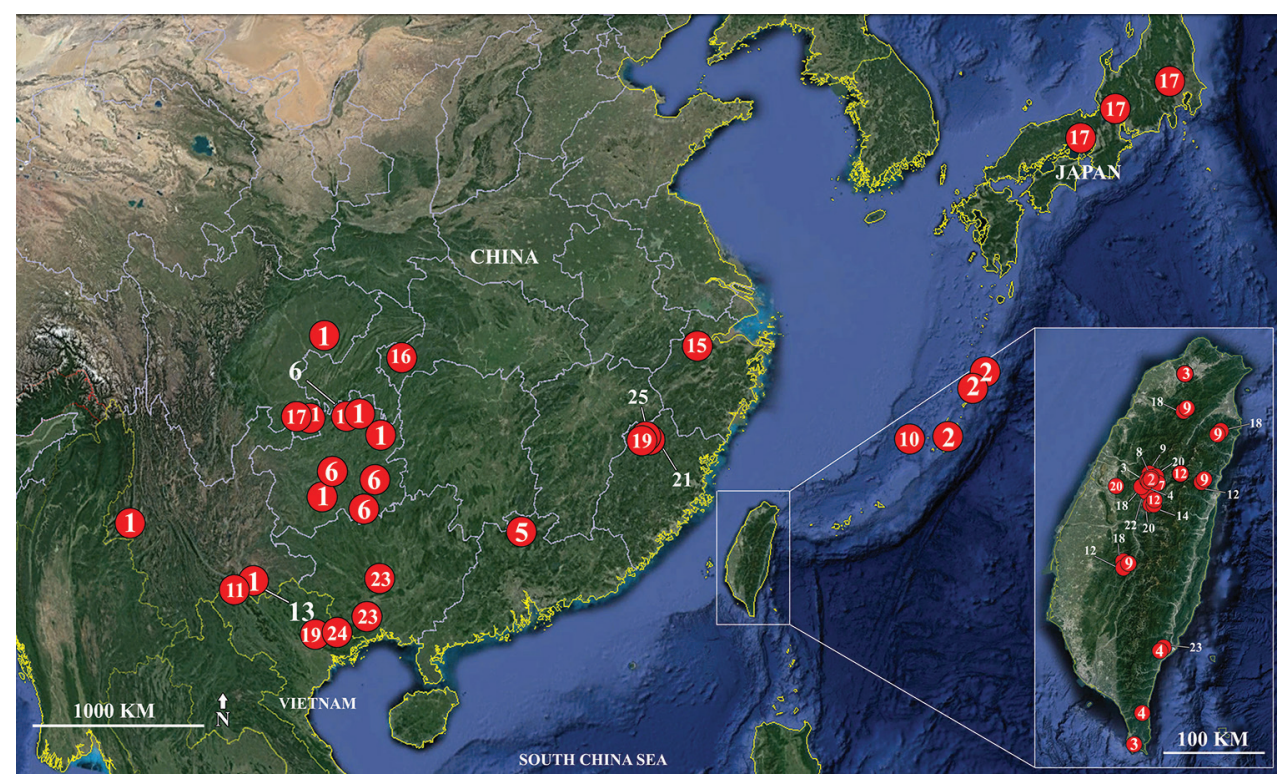

Figure 9. Distribution records of the species from genus Betacixius I B. bispinus Zhang \& Chen, 20112 B. brunneus Matsumura, 19143 B. clypealis Matsumura, 19144 B. delicatus Tsaur \& Hsu, 19915 B. euterpe Fennah, 19566 B. flagellihamus Zhang \& Chen, 20117 B. flavovittatus Hori, 1982 8 B. fuscus Tsaur \& Hsu, 19919 B. herbaceus Tsaur \& Hsu, 1991 IO B. kumejimae Matsumura, 1914 II B. latissimus sp. nov. I2 B. maculosus Tsaur \& Hsu, 1991 I3 B. maguanensis sp. nov. I4 B. michioi Hori, 1982 I5 B. nelides Fennah, 1956 I6 B. nigromarginalis Fennah, 1956 I7 B. obliquus Matsumura, 191418 B. ocellatus Matsumura, 1914 I 9 B. pallidior Jacobi, 194420 B. rinkihonis Matsumura, 1914 21 B. robustus Jacobi, 194422 B. shirozui Hori, 198223 B. sparsus Tsaur \& Hsu, 199124 B. tonkinensis Matsumura, 191425 B. transversus Jacobi, 1944.

and tree leaves, at elevations up to $2600 \mathrm{~m}$. Unfortunately, there is no knowledge of what plants these planthoppers really feed on, except that B. flagellihamus was collected on Quercus sp. (Fagaceae), which might be the plant on which it feeds.

Based on published data and our field surveys, the distribution of Betacixius is mostly restricted to the Oriental parts of China, Japan, and Vietnam, with B. obliquus Matsumura, 1914, occurring in both Palaearctic and Oriental regions, as the only exception (Fig. 9).

The regional studies on Betacixius in China are not equally efficient at finding species. To date, 23 species are known from China, of which about half that number (12 species) are known from Taiwan and only 12 species have been described from southern mainland China, which is much broader and more variant in ecological complexity compared with Taiwan. Therefore, we believe that additional comprehensive field surveys will find that the diversity of Betacixius in mainland China is doubtlessly richer. 


\section{Acknowledgements}

We are grateful to the specimen collectors for their hard work in the field collections. This work was supported by the National Natural Science Foundation of China (no. 31472033), the Program of Excellent Innovation Talents, Guizhou Province (no. 20154021), the Program of Science and Technology Innovation Talents Team, Guizhou Province (no. 20144001), the International Cooperation Base for Insect Evolutionary Biology and Pest Control (no. 20165802), the Science and Technology Project of Guiyang (no. [2017]5-25), and the Science-Technology Program of Guizhou Tobacco Company Zunyi Branch (no. [2018]11).

\section{References}

Bourgoin T (1987) A new interpretation of the homologies of the Hemiptera male genitalia, illustrated by the Tettigometridae (Hemiptera, Fulgoromorpha). Proceedings $6^{\text {th }}$ Auchenorrhyncha Meeting, Turin, Italy, 7-11 September 1987, 113-120.

Bourgoin T (1993) Female genitalia in Hemiptera Fulgoromorpha, morphological and phylogenetic data. Annales de la Société Entomologique France 29(3): 225-244.

Bourgoin T, Wang RR, Asche M, Hoch H, Soulier-Perkins A, Stroiński A, Yap S, Szwedo J (2015) From micropterism to hyperpterism: recognition strategy and standardized homology-driven terminology of the forewing venation patterns in planthoppers (Hemiptera: Fulgoromorpha). Zoomorphology 134: 63-77. https://doi.org/10.1007/s00435-014-0243-6

Bourgoin T (2020) FLOW (Fulgoromorpha Lists on the Web): a world knowledge base dedicated to Fulgoromorpha. Version 8, updated 30 March 2020. http://hemiptera-databases. org/flow/ [Accessed on: 2020-4-2]

Emeljanov AF (2002) Contribution to classification and phylogeny of the family Cixiidae (Hemiptera, Fulgoromorpha). Denisia 04: 103-112.

Fennah RG (1956) Fulgoroidea from Southern China. Proceedings of the California Academy of Science (4th series) 28: 441-527.

Hori Y (1982) The genus Betacixius Matsumura, 1914 (Homoptera: Cixiidae) of Formosa. In: Satô M, Hori Y, Arita Y, Okadome T (Eds) Special Issue to the Memory of Retirement of Emeritus Professor Michio Chûjô. Association of the Memorial Issue of Emeritus Professor M. Chûjô C/O Biological Laboratory, Nagoya Women's University, Nagoya, 175-182.

Jacobi A (1944) Die Zikadenfauna der Provinz Fukien in Südchina und ihre tiergeographischen Beziehungen. Mitteilungen der Münchner Entomologischen Gesellschaft 34: 5-66.

Matsumura S (1914) Die Cixiinen Japans. Annotationes Zoologicae Japonenses 8: 393-434.

Tsaur SC, Hsu TC, Stalle JV (1991) Cixiidae of Taiwan, Part V. Cixiini except Cixius. Journal of Taiwan Museum 44: 1-78.

Zhang P, Chen XS (2011) A checklist and key to species of the genus Betacixius Matsumura (Hemiptera: Fulgoromorpha: Cixiidae) with descriptions of two new species from Guizhou province, China. Florida Entomologist 94(1): 48-56. https://doi.org/10.1653/024.094.0107 